Article

\title{
Reduction of Map Information Regulates Visual Attention without Affecting Route Recognition Performance
}

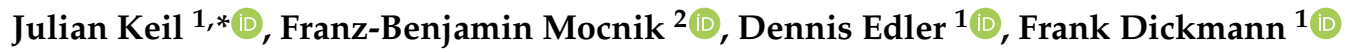 \\ and Lars Kuchinke ${ }^{3}$ \\ 1 Geography Department, Cartography, Ruhr University Bochum, 44801 Bochum, Germany; \\ Dennis.Edler@ruhr-uni-bochum.de (D.E.); frank.dickmann@rub.de (F.D.) \\ 2 Institute of Geography, Heidelberg University, 69120 Heidelberg, Germany; mocnik@uni-heidelberg.de \\ 3 Methodology and Evaluation, International Psychoanalytic University, 10555 Berlin, Germany; \\ lars.kuchinke@ipu-berlin.de \\ * Correspondence: Julian.Keil@rub.de; Tel.: +49-2324-32-23662
}

Received: 27 September 2018; Accepted: 28 November 2018; Published: 30 November 2018

\begin{abstract}
Map-based navigation is a diverse task that stands in contradiction to the goal of completeness of web mapping services. As each navigation task is different, it also requires and can dispense with different map information to support effective and efficient wayfinding. Task-oriented reduction of the elements displayed in a map may therefore support navigation. In order to investigate effects of map reduction on route recognition and visual attention towards specific map elements, we created maps in which areas offside an inserted route were displayed as transparent. In a route memory experiment, where participants had to memorize routes and match them to routes displayed in following stimuli, these maps were compared to unmodified maps. Eye movement analyses revealed that in the reduced maps, areas offside the route were fixated less often. Route recognition performance was not affected by the map reduction. Our results indicate that task-oriented map reduction may direct visual attention towards relevant map elements at no cost for route recognition.
\end{abstract}

Keywords: cognitive cartography; empirical cartography; spatial cognition; volunteered geographic information; landmarks; map pictograms; route memory; recognition; story telling

\section{Introduction}

In today's world, human life is accompanied by high mobility. Traveling to unfamiliar regions has become simple and cheap, increasing the need for navigation in unfamiliar environments. Geographic information in the form of maps or navigation systems is thus of increasing importance. Modern web mapping services such as OpenStreetMap, an example of Volunteered Geographic Information (VGI) [1], and Google Maps provide fairly accurate geographic information at no cost [2,3]. In the era of smartphones and mobile internet, these map distributers can be used virtually everywhere. Additionally, navigation apps can support wayfinding in unfamiliar environments.

Besides navigation, maps are often used for telling stories. Television, films, social media, travelogues, newspapers, and audio books are ubiquitous examples of media used for conveying stories, demonstrating their high social relevance. As stories often have a spatial component-things exist and happen in space-maps can be used for this purpose. Today, maps can easily be extended with other valuable media, such as texts, audio, and video [4-6]. This helps to widen the number of map genres and to adapt the needs of a spatial story [7].

In both cases-navigation and storytelling-it can be advantageous to focus on the essential information. Many maps, especially topographic maps, are task-independent. Such maps are created 
to represent the real environment in a most complete way. Thus, they display all information that complies with the categories provided in the legend or an ontology. As an example, one expects a city map to contain all streets in the depicted area. Such information might, however, be irrelevant to the user when performing a certain task. Leaving out unneeded information can have several consequences. One might assume that reduced maps which do not display all information provide fewer distractions when navigating. Also, the user of a reduced map might get an impression that the map is, in fact, incomplete. As a consequence, the user develops an open-world assumption. Assuming gaps or errors in the map opens the possibility of more flexible use and might aid the map user when telling a story or being confronted with inaccurate map information. Despite of the assumed usefulness of reduced maps, potential positive or negative consequences have only been examined in part so far $[7,8]$.

In this article, we examine in which way the absence of information in a map used for a navigation task influences our cognition. A reduced map provides less information that distracts the user, but also less information that provides context to the relevant parts of the map. We focus on the following two research questions.

RQ1. Does the reduction of map elements towards only the informative parts of the map affect route memory?

RQ2. Does the reduction of the represented content of a map shift visual attention towards a displayed route?

For answering these questions, participants were asked to memorize a route in a reduced map. Thereafter, it was tested how well the participants performed at recognizing the shape of the route. These results were set into context by a comparison to recognition performance when using a conventional nonreduced map.

\section{Background}

Both digital maps and navigation systems enclose a tradeoff based on their design. As mentioned before, maps are usually task-independent and strive for completeness. Additionally, they allow users to obtain survey knowledge of their surroundings [9]. However, they also contain a lot of information that is irrelevant for specific navigation tasks. Studies have shown that the degree of visual complexity in a map affects performance in map-based memory tasks [10-12]. While Kuchinke et al. [10] showed that topographic detail improved recognition performance of object locations in maps, Edler et al. [11-13] found that improvements of memory performance based on the presentation of additional map elements become less noticeable at exceedingly high levels of map complexity. Given that visual complexity of stimuli can increase the cognitive load of the perceiver [14], existence of a tipping point can be presumed where the amount of displayed information is no longer helpful for map-based memory tasks and distracts from relevant visual elements. Navigation apps on the other hand are highly task-oriented and, as usual for location-based services (LBS), the displayed content depends on the context (current position). They support efficient wayfinding in unfamiliar environments, but they usually visualize only a narrow area around the position of the user. This can impair orientation and route memory, as distant global landmarks are not displayed [15]. Additionally, the lack of active interaction with the environment prevents the acquisition of spatial knowledge about the environment [16]. An ideal navigation aid would therefore combine the strengths of digital maps and navigation systems - fast and efficient wayfinding, limited cognitive load, focus on relevant map elements, and a survey view of the environment that supports the formation of survey knowledge $[9,17]$.

In our experiment, we examine the use of reduced maps adapted to specific use cases in order to overcome the tradeoffs of maps and navigation systems in wayfinding tasks. When people want to communicate a route without external aids, they often use sketch maps, hand-drawn maps that show the whole route at once, but leave out most peripheral elements shown in a "classical" map. 
They are usually incomplete [18-20], i.e., they only contain roads and road sections alongside the route, and landmarks at decision points [21]. Such sketch maps are a graphical representation of the task-oriented cognitive map of their creators [22]. These sketch maps seem to be perfectly reduced to tell the story of how to follow the route to aid route learning and navigation. Therefore, reducing maps based on sketch map pattern may improve route memory performance.

Based on this assumption, we investigate the possibility to limit the complexity of maps and the consequential effects on cognitive load. The common cartographic approach for reducing map complexity is generalization. Generalization describes the process of simplifying boundaries of map elements and removing seemingly less relevant elements [23]. However, map users may not recognize task-oriented map generalization instantly, certainly not what elements have been removed. Consequentially, an open-world assumption will not be generated before the map user is confronted with a confusing mismatch of the current position and its map representation, e.g., if a small road is not displayed in the map. Therefore, we apply a different approach by displaying areas offside of the route transparent. Given that visual attention is affected by the transparency of stimuli [24], transparent areas offside the route could shift the visual attention of the user towards relevant map elements, namely the area around the route, while a nongeneralized survey view of the environment is still available. Eye fixations are reported to indicate visual attention and are therefore commonly used to assess visual attention towards specific stimulus areas [25-27]. Consequently, investigating eye fixations on maps using an eye tracker could unveil whether displaying specific map areas transparent shifts visual attention towards other non-transparent map areas.

If all elements in a map offside a displayed route are invariably displayed transparent, it needs to be considered that this may also deteriorate positive aspects of a survey map. Especially landmarks are highly relevant for orientation, navigation and the formation of cognitive maps $[28,29]$ and are expected to be important elements of navigation stories. Therefore, the display format of landmark pictograms can affect navigation and route recognition performance [30]. Landmark pictograms in OpenStreetMap and Google Maps are displayed based on the selected scale of the map. When a small scale is selected, only few of the deposited landmark pictograms are displayed. At the largest scale, all deposited landmarks are displayed. Removing or adding such map elements based on map properties as scale would force the user to rely on other map elements for route recognition, which may in turn impair recognition performance. In order to assess whether the task-specific reduction of maps and the display of landmark pictograms affect route perception and recognition, we test the following hypotheses in our experiment.

Hypotheses 1 (H1). Displaying areas offside of the route transparent does not impair route recognition performance.

Hypotheses 2 (H2). Displaying areas offside of the route transparent shifts visual attention towards the route.

Hypotheses 3 (H3). Adding or removing landmark pictograms after the route has been memorized impairs route recognition performance.

\section{Methods}

The study was conducted in accordance with the Declaration of Helsinki. The experimental design has been controlled by the ethics committee of the Faculty of Geosciences at the Ruhr-University Bochum and was classified as ethically acceptable (13 July 2018).

\subsection{Participants}

The study sample comprised 69 geography students (30 females, 39 males) of the Ruhr-University Bochum with normal or corrected vision and no neurological diseases. Their age range was between 18 and 37 years $(M=23.07 ; S D=3.45)$. Participation was rewarded with a payment of 5 EUR. 


\subsection{Materials}

Participants were sorted into two experimental between-subject conditions (standard vs. reduced maps) with the same distribution of sexes in each. For both conditions, six maps (study maps) containing a route marked with a red line, a green starting point indicator, and a red destination indicator were built (Figure 1). The base maps were extracted from OpenStreetMap (OSM) in a scale of 1:10,000 and represented the same six regions in both conditions. All maps showed European urban regions selected to prevent high familiarity of the participants with the displayed regions. In the first condition (reduced maps), all map areas with a distance of more than 10 pixels to the route were displayed transparent (alpha value $=12$ ). In the second condition (standard maps), no map areas were displayed transparent.

Two variants of each map in both conditions were generated. One variant contained OSM landmark pictograms close to each route diversion as well as at additional random positions in the map. The used landmark pictograms were selected from the OSM landmark pictogram repository based on their salience and meaningfulness [31]. Twenty landmarks with moderate salience and meaningfulness were chosen in order to prevent extensive attention towards single landmark pictograms with higher salience [32] or higher meaningfulness [33]. For each landmark position in the study maps, one of these 20 landmark pictograms was selected at random. The second study map variant contained no landmark pictograms. After the route was inserted and all street names were removed, maps were exported in a size of $30 \times 20 \mathrm{~cm}(1063 \times 709$ pixels $)$. See examples for both experimental conditions and variants in Figure 1.
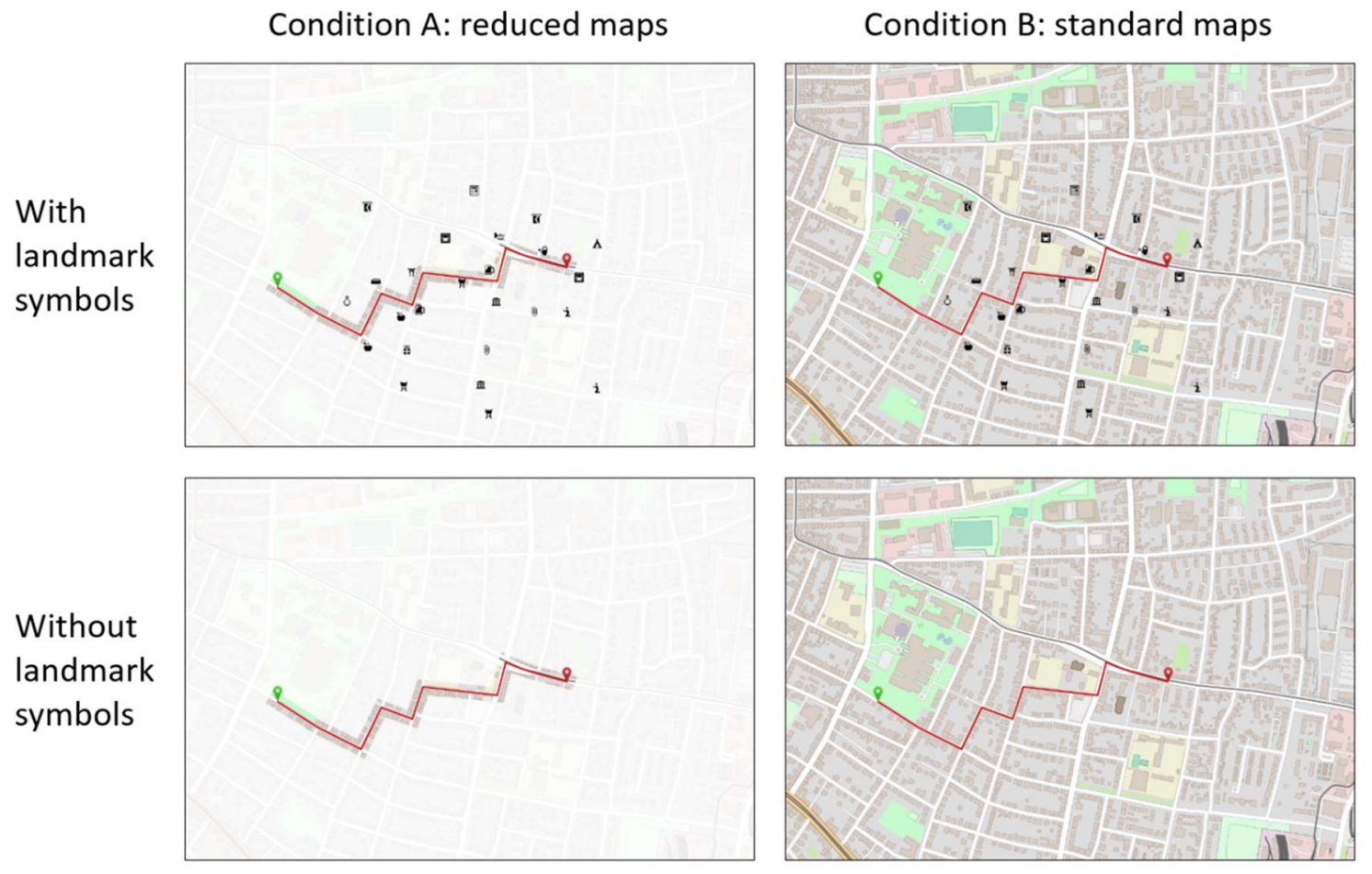

Figure 1. Study map conditions and variants. According to their experimental condition, participants saw either six reduced or six standard study maps. Participants from both conditions saw three maps with landmark pictograms and three maps without landmark pictograms.

Additionally, four types of recognition stimuli (examples in Figure 2) were generated for each of the six study maps to test whether participants could recognize the correct route shape among incorrect route shapes. These stimuli had the same size as the study maps. They also contained a route marked with a red line, a green starting point indicator, and a red destination indicator. The recognition stimuli showed no map, but a blank white background. Per study map, at least 
one of the four corresponding recognition stimuli contained the same route shape as the study map (correct route). The other recognition stimuli contained altered versions of the original route shape (incorrect route). The random amount of correct route shapes was intended to prevent that participants recognize a constant proportion of correct and incorrect routes, as it would enable them to anticipate whether the following stimulus shows a correct route if all correct or incorrect route shapes have already been shown. Similar to the study maps, two variants of each recognition stimulus were generated. One variant contained the same landmark pictograms as the version with landmarks of their corresponding study map. The second variant contained no landmark pictograms. All correct and incorrect routes contained six route diversions. Route diversions of incorrect routes were also placed close to landmark pictograms positions (if the stimulus contained landmark pictograms), but different pictogram positions than the ones used for the correct route. In the case of incorrect routes in stimuli without landmarks, route diversions were placed close to the positions of landmark pictograms in their correspondent study map stimulus that included landmarks. In both experimental conditions (reduced and standard maps), the same recognition stimuli were used.

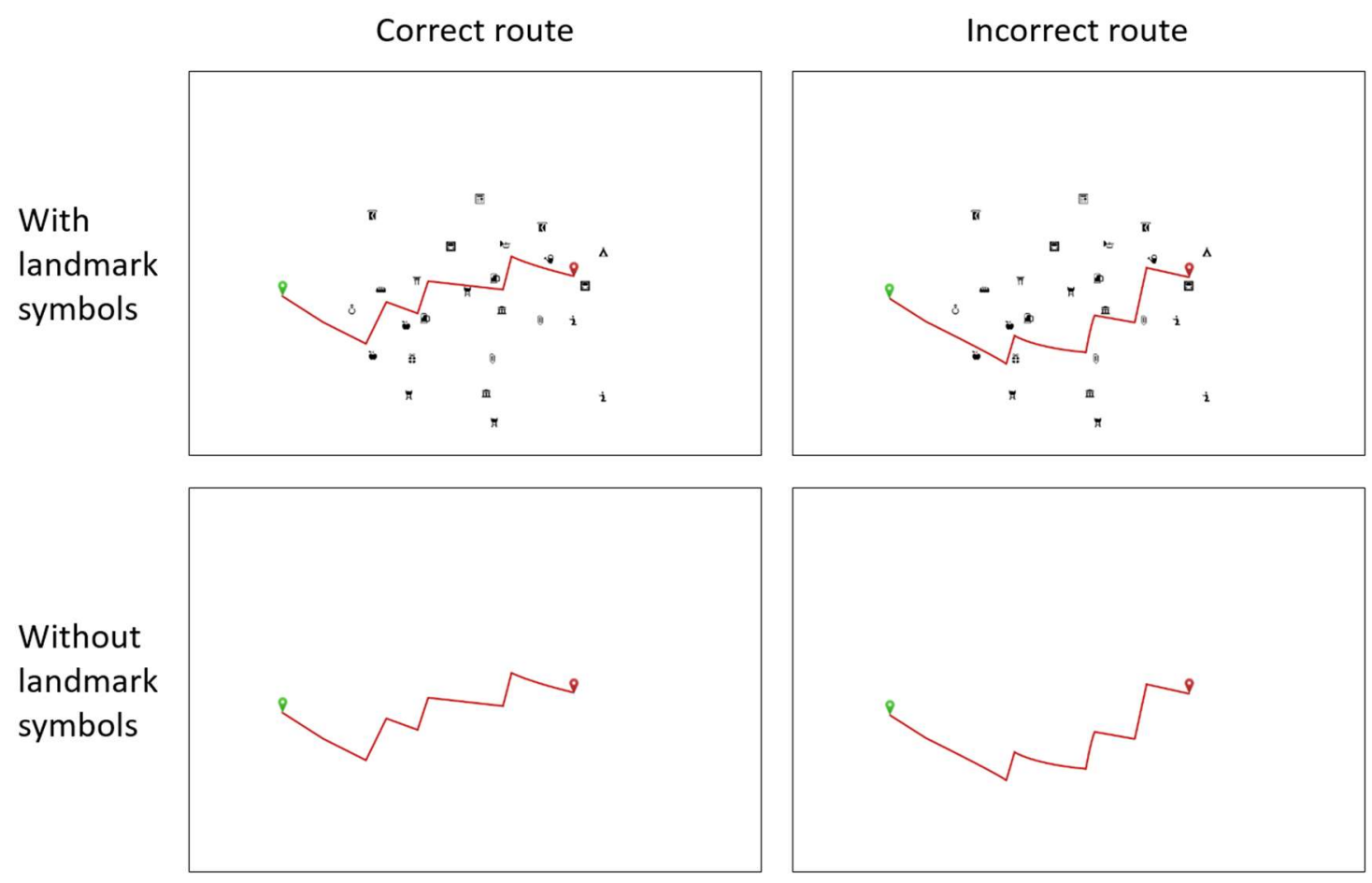

Figure 2. Recognition stimulus variants. After each study map, four recognition stimuli were shown to the participants. At least one of these stimuli contained the same route as the study map. The rest contained slightly changed route shapes. Whether landmark pictograms were displayed in a recognition stimulus was determined at random.

\subsection{Procedure}

In order to prevent response biases, no information about the study purpose was given to the participants before or during study participation [34,35]. They were told that information concerning the study purpose would be provided after the experiment. Before the experiment started, the procedure was explained and the participants gave informed consent. Hereafter, they took a seat in front of a Tobii TX-300 ( $300 \mathrm{~Hz}, 23$ inches) eye-tracker monitor that was used to visualize the stimuli. The distance between the eyes and the monitor was $65 \mathrm{~cm}$.

The experiment consisted of a practice trial and six experimental trials. At the beginning of each trial, a study map was shown for $30 \mathrm{~s}$. During this time, participants had to memorize the route displayed in the map. Participants were presented only maps that belonged to the experimental 
condition a participant was assigned to (reduced maps or standard maps). Three of these six study maps shown in the experimental trials were randomly selected to display landmarks while the other 3 maps did not contain landmarks (i.e., within-subject factor 'study map landmark' yes or no). The presentation order of the six selected study maps was randomized. After every $30 \mathrm{~s}$ study phase, the four recognition stimuli belonging to the previously shown study map were presented successively, each for eight seconds. The presentation order and the variant selection of each recognition stimulus (with or without landmarks) were randomized. The matching of study maps and recognition stimuli with and without landmarks allowed to compare recognition performance between conditions in which landmarks were shown only in the study phase, only in the recognition phase, in both phases or in none of them. After every recognition stimulus presentation, participants had to answer whether the route displayed in the previous recognition stimulus had exactly the same shape as the route displayed in the last study map. The answers were given by pressing one of two keyboard keys labeled with "yes" and "no".

\subsection{Measures}

\subsubsection{Recognition Performance}

Performance in the recognition task was assessed according to the signal detection theory [36] in the form of hits, misses, correct rejections, and false alarms. If the route shape in a recognition stimulus matched the route shape in the study map (old stimuli), participants could either correctly state a match (hit) or wrongly state a mismatch (miss). If the two route shapes did not match (new stimuli) participants could either correctly state a mismatch (correct rejection) or wrongly state a match (false alarm). Because of the redundancy in these measures, only the hits and correct rejections were investigated in the statistical analyses. The misses and false alarms were merely used to calculate d', an additional recognition performance measure based on all four response types. The benefit of $\mathrm{d}^{\prime}$ is that it puts correct signal detection (hits and correct rejections) and noise responses (misses and false alarms) in proportion [36,37]. The $\mathrm{d}^{\prime}$ value increases if the ratio of hits and correct rejection increase. It decreases if the ratio of misses and false alarms increase. This allows to make statements about the sensitivity of how well participants discriminate old from new stimuli. For information about d' calculation see Macmillan \& Creelman [38].

\subsubsection{Visual Attention}

Eye fixation measures have been reported to be related to mental processing of visual stimuli [27,39]. Therefore, average fixation duration and fixation count inside predefined Areas-of-Interest (AOIs) were used as measures for visual attention. Two AOIs were placed in each study map of both experimental conditions (reduced and complete maps). The first AOI covered the displayed route and the area that was not displayed transparent in the first experimental condition (reduced maps). The second AOI covered all areas that were not covered in the first AOI (areas offside the route). This enabled us to compare the visual attention towards the displayed route (AOI 1) and other map areas (AOI 2) between the two experimental conditions. In eye-tracker studies, completeness of gaze data is an important quality criterion. Droopy eyelids and positioning the head outside of the tracking area of the eye-tracker may lead to gaze data loss [40]. Such data loss may cover up important information about visual attention towards specific areas of a stimulus. Therefore, eye-tracker recordings with massive gaze data loss should be removed from analysis. According to Bojko [40], a gaze data loss threshold between $10 \%$ and $30 \%$ may be selected. Based on this suggestion, we defined a threshold of $25 \%$. 


\subsection{Statistics}

As our method generated multiple measurements per participant and item, and as visual inspections revealed that the response variables were skewed, we chose a generalized estimating equation (GEE) model for our first statistical analysis. The GEE model is an extended version of the generalized linear models. It can handle correlations of clustered data (repeated measures) and non-normally distributed response data [41] and can be seen as a robust alternative for multifactorial ANOVA models. Recognition performance (hits and correct rejections) main effects were calculated for the between-subject factor (reduced/standard map) and the two within-subject factors (landmarks/no landmarks in the study maps and the recognition stimuli). Additionally, interaction effects between the three factors were assessed.

Given that $\mathrm{d}^{\prime}$ values put correct and incorrect responses into proportion, calculating $\mathrm{d}^{\prime}$ requires aggregation of hits, misses, correct rejections, and false alarms across participants and specific conditions. This undermines the benefit of the GEE model to handle correlations of multiple responses from the same subjects at the level of single items. The same is true for the visual attention measures, which generated only one fixation count and average fixation duration value per participant and study map. In addition, the fixation data did not follow a Gaussian distribution. Therefore, the nonparametric Mann-Whitney $\mathrm{U}$ test was used to compare $\mathrm{d}$ ' and eye fixations data between the two map conditions (reduced/standard map). For the examination of the within-subject effects of landmarks (in study or recognition stimuli), $\mathrm{d}^{\prime}$ values and fixation data were analyzed with Wilcoxon signed-rank tests. Additionally, the Wilcoxon signed-rank test was used to compare fixation counts and average fixation durations between the route AOI and the AOI offside the route separately for each map condition (reduced/standard map).

Unfortunately, not all participant data could be used for the fixation analysis. For six participants, the eye-tracker could not be calibrated successfully. Seven other participants exceeded the predefined threshold of $25 \%$ gaze data loss. This reduced the sample size of the eye-tracking analyses from 69 to 56 participants.

\section{Results}

The GEE model that analyzes the hits found no statistically significant differences in the between-subjects condition (study map condition, $p=0.757$ ), nor regarding the within subjects conditions (study map landmark condition, $p=0.607$; recognition landmark condition, $p=0.324$ ). Also, no significant interaction effect was found in the GEE (all $p$-values $>0.089$ ). In contrast, the examination of the correct rejections revealed a significant effect of the study map landmark condition $(p=0.028)$. Correct rejection values were significantly higher when no landmarks were shown in the study map (see Figure 3, $\mathrm{M}_{\text {noLandmarks }}=0.931, \mathrm{M}_{\text {Landmarks }}=0.882$ ). No other effect regarding the correct rejections was significant (study map conditions, $p=0.956$; recognition landmark condition, $p=0.457$ ). No significant interaction effects were observed in the correct rejection data (all $p^{\prime} \mathrm{s}>0.228$ ).

The examination of the discrimination performance measure $d^{\prime}$ revealed no differences between the participant groups (study map condition, $\mathrm{U}=641, p=0.583$ ), the within-subjects effects of study map landmark condition $(\mathrm{W}=1344, p=0.416)$, or the recognition landmark conditions $(\mathrm{W}=1182$, $p=0.879)$. 


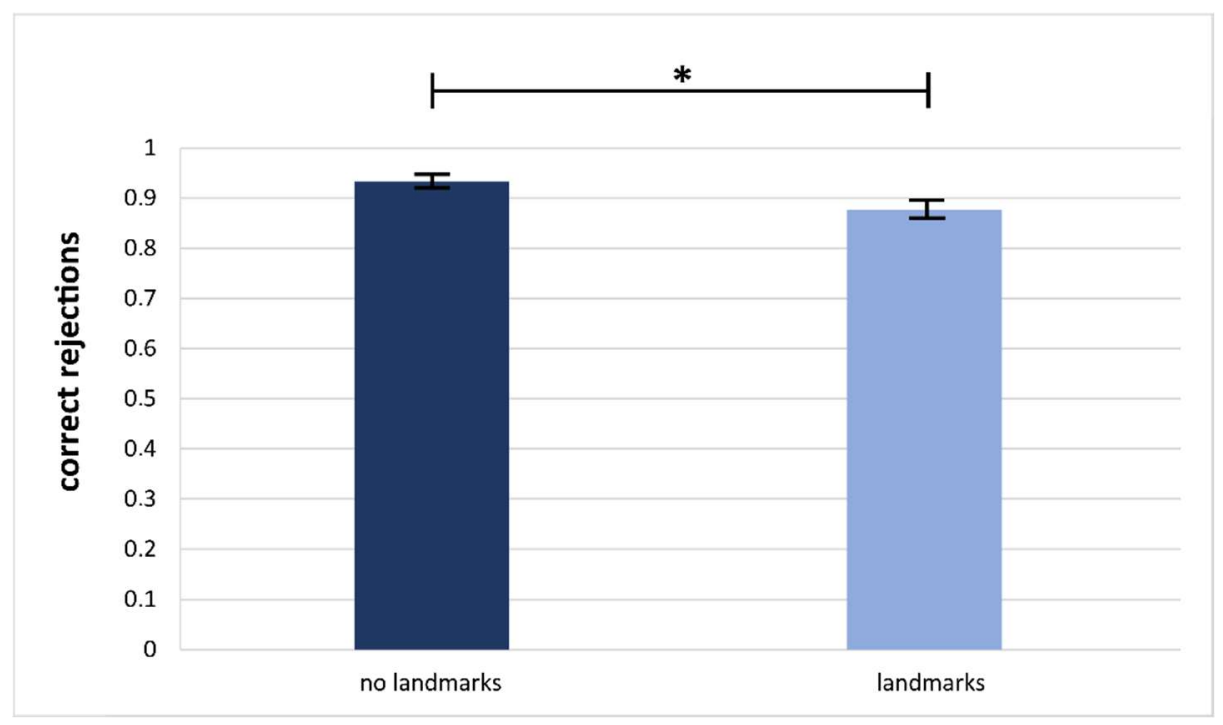

Figure 3. Mean correct rejection difference between the study map landmark conditions. The mean correct rejection of incorrect routes was higher when no landmarks were shown in the study map $\left(\mathrm{M}_{\text {noLandmarks }}=0.931, \mathrm{M}_{\text {Landmarks }}=0.882, p=0.028\right)$.

This picture is different, when it comes to the eye-tracking data. Here, the fixation count differed significantly between the route AOI and the offside route AOI (see Figure 4) both in the reduced maps $\left(\mathrm{M}_{\text {RouteAOI }}=42.867, \mathrm{M}_{\text {OffsideRouteAOI }}=2.724, \mathrm{~W}=1, p<0.001\right)$ and the standard maps $\left(\mathrm{M}_{\text {RouteAOI }}=45.506, \mathrm{M}_{\text {OffsideRouteAOI }}=36.136, \mathrm{~W}=88, p=0.015\right)$. The differences of average fixation durations between the route $\mathrm{AOI}$ and the offside route $\mathrm{AOI}$ in the reduced maps $\left(\mathrm{M}_{\text {RouteAOI }}=0.379, \mathrm{M}_{\text {OffsideRouteAOI }}=0.332, \mathrm{~W}=22, p=0.030\right)$ and in the standard maps $\left(\mathrm{M}_{\text {RouteAOI }}=0.350, \mathrm{M}_{\text {OffsideRouteAOI }}=0.297, \mathrm{~W}=11, p<0.001\right)$ were also statistically significant. The fixation count on the AOI covering the areas offside the route differed significantly between the two study map conditions $\left(\mathrm{M}_{\text {Reduced }}=2.724, \mathrm{M}_{\text {Complete }}=36.136, \mathrm{U}=0, p<0.001\right)$, but not for the route AOIs $\left(\mathrm{M}_{\text {Reduced }}=42.867, \mathrm{M}_{\text {Complete }}=45.506, \mathrm{U}=350, p=0.496\right)$. Average fixation duration differences on the AOI offside the route $(\mathrm{U}=224, p=0.586)$, as well as average fixation duration $(\mathrm{U}=438, p=0.454)$ on the route AOI did not differ significantly between the two study map conditions.
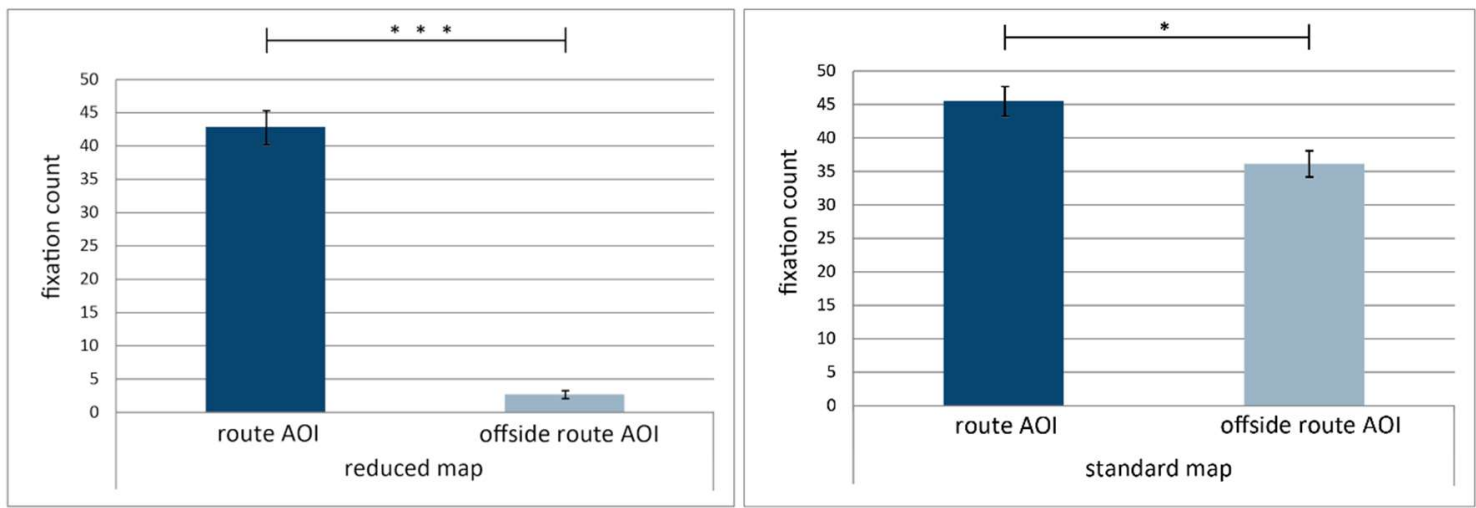

Figure 4. Fixation count differences between the route AOI and the offside route AOI for both map conditions. In both map conditions, fixation counts on the route AOI were significantly higher than fixation counts on the AOI offside the route. In the reduced maps $\left(\mathrm{M}_{\text {RouteAOI }}=42.867\right.$, $\mathrm{M}_{\text {OffsideRouteAOI }}=2.724, \mathrm{~W}=1, p<0.001$ ), the difference was larger than in the standard maps $\left(\mathrm{M}_{\text {RouteAOI }}=45.506, \mathrm{M}_{\text {OffsideRouteAOI }}=36.136, \mathrm{~W}=88, p=0.015\right)$. 


\section{Discussion and Conclusions}

\subsection{Discussion}

The experiment presented above provides insights into how map reading is affected by reducing the amount of information displayed in a map. In the following, we discuss implications for the design of maps used in navigation tasks, among others, in respect to landmarks displayed on the map.

Regarding the general research question, two main results were obtained: Learning a route on a complete map display or with a reduced map that displays areas offside the route transparent did not affect route recognition memory performance and, in agreement with our first hypothesis, no behavioral differences were revealed between participants in the complete study map condition or the reduced study map condition. In other words, reducing the map display to only the significant 'story' of a map (in the present study the route) did not improve nor deteriorate performance in a route learning paradigm. Thus, we found some evidence that route learning does not depend on the distant or uninformative map regions but indeed on the detail (and also likely on the landmarks, see below) in the close neighborhood of the to-be-learned route. At first glance, these results seem surprising, as there is no evidence of an advantage in information processing when participants are forced to focus on the essential map information [7]. Besides the possibility of methodological factors contributing to a null effect (like a small number of items in a condition) and the logical difficulties when deriving inverse assumptions, the results still indicate that it is possible to reduce the map display without further costs when the task is to recognize a route. This is in agreement with the findings of earlier studies [8,42]. Meilinger et al. [8] showed that reduction of map complexity did not affect orientation and even improved wayfinding performance in larger areas. $\mathrm{O}^{\prime} \mathrm{Neill}$ [42] also found that high map complexity may negatively affect wayfinding performance. These studies together with the present results demonstrate that simplified or reduced maps may support route-based tasks just as well as a standard map.

The second result refers to the eye-tracking data that is indicative of changes in visual attention. Here reduced maps clearly led to fewer fixations on map areas offside the route during the study map condition. This supports our second hypothesis and complements the results of Meilinger et al. [8], who found that reducing the complexity of a map also reduced the time that participants needed for studying the map. The difference of fixation counts between the two map conditions is not visible for the close neighborhood of the route (i.e., in the route AOI). Thus, a reduction of visual input led to differences in how participants look at the map; not that they spent more fixations on the route (and its neighborhood) - the pattern changed for the whole map with proportionally more fixations directed towards the route. As stated before, eye fixations are assumed to indicate mental processing of stimuli [27,39]. Even though the higher proportion of fixations towards the route did not directly contribute to better route memory performance in the present study, it seems likely that deeper processing explains to some extend why a reduced map does not lead to a drop of memory performance. Summing up, we can partially confirm our hypothesis that the reduction of map complexity shifts attention towards the route, as proportionally to all fixations on the map, more fixations were targeted on the route. It seems that users of the reduced map were less prone to distractions by map elements that are irrelevant to route memory.

Unexpectedly, neither the display of landmark pictograms in the study map, nor removing or adding landmark pictograms in the recognition stimuli affected route recognition performance (as it regards the learned old items). Displaying landmark pictograms along the route did not improve route recognition performance, which stands in contrast to the findings of Tom \& Denis [30], who showed that the display of landmark pictograms in maps improves route recognition. But displaying landmarks during memory encoding reduced the number of correct rejections. Hence, participants made more false alarms, i.e., falsely recognized a new route as being learned if landmarks are displayed during study. While the performance on old items (hits) was not affected by the availability of landmarks during study or recognition, the significant effect for new routes reveals that landmarks are part 
of the mental representation. Learning on a map with landmarks leads to higher false alarm rates. Thus, these data are in line with findings by Franke \& Schweikart [43], who demonstrate a positive effect of landmark display on the formation of cognitive maps. They also partially confirm our third hypothesis that adding or removing landmark pictograms after a route has been learned negatively affects recognition performance.

\subsection{Limitations and Proposed Further Research}

The presented study should be regarded as a first step towards the development of more user-friendly and task-oriented maps. Additional research in this area could give clear instructions about how maps used for navigation and route learning tasks should be designed.

Possible effects of landmark pictogram display on route recognition may have been inhibited by the experimental design. The availability of landmark pictograms in both the study maps and the recognition stimuli was completely randomized. Therefore, participants may have learned after a few trials that they cannot rely on learning the route based on its relative position to landmark pictograms, as these may not be available in the recognition phase. In order to assess the effect of landmark pictograms on route recognition more accurately, availability of landmark pictograms should be a between-subject factor, or at least consistent across a single trial including study phase and recognition phase. If the experiment would be adjusted accordingly, we would expect to find significant results comparable to previous studies [8,42].

The lack of significant results in the recognition task implies that it might have been too easy. Reducing the presentation time may lead to more distinct recognition performance differences between the experimental conditions. Furthermore, requesting participants to respond during stimulus presentation instead of afterwards would allow to use time on task as a measure for task efficiency.

Additional levels of transparency (alpha levels) should be investigated. When lower transparency levels of areas offside the route are selected, visual attention should approach the pattern of standard maps as the map complexity increases. If map areas offside the road are on the other hand completely removed, a radical change of visual attention and recognition performance may occur. In this case, visual attention would be expected to only focus on the route and potentially displayed landmarks, as no other objects would be available offside the route. The question remains whether route recognition performance would still remain stable with such radical map reduction, as the amount of visual anchors inside the map would be massively reduced to the close neighborhood of the to-be-learned route.

There are additional aspects beyond route recognition performance and visual attention that could be affected by map reduction. As shown by Dutta-Bergman [44], trust in information is affected by its completeness. This raises the question whether map reduction can affect the credibility of the map, because users expect relevant information to be missing. Obviously missing map elements could on the other hand also, as mentioned in the introduction, lead to an open-world assumption. Being aware of the incompleteness of the map could lead to a higher flexibility when representations of real-world objects in the map are inaccurate or unexpectedly missing [7]. Therefore, deliberately activating an open-world assumption via directly recognizable map reduction may be advantageous if the accuracy and completeness of map information cannot be guaranteed. Effects of map reduction and the activation of an open-world assumption on trust in the displayed content and the use of inaccurate and incomplete information could be investigated by confronting people with deliberately incomplete and inaccurate maps of familiar environments.

Additional focus might also be set on the impact of storytelling on route memory and navigation performance. As Bellezza et al. [45] demonstrated, generating story mnemonics with to-be-learned objects improves memory performance. Such a storytelling approach could also be incorporated into the present study design: In the landmark condition, there was a landmark pictogram close to each decision point of all routes. Effects of storytelling on route recognition could be investigated by comparing results of our study design with maps where the same landmark symbol is shown at each decision point of all routes. If the effects of map reduction and landmark placement on navigation 
performance and map credibility are better understood, maps could be designed in a way that supports wayfinding efficiently and effectively without sacrificing the users' trust.

\subsection{Summary}

The present study was targeted at assessing the effects of map reduction and landmark display on route recognition and visual attention. We were able to demonstrate that reducing a map by displaying map areas offside a route transparent does not affect route recognition performance. However, reducing the map shifted proportionally more fixations towards a displayed route. Presenting incongruent information by removing or adding landmark pictograms after a route had been memorized only affected recognition performance of new stimuli (correct rejections and false alarms), but not of old stimuli (hits and misses), which we argued to be affected by our experiment design. Overall, our findings indicate that task-oriented reduction of map complexity is a feasible approach to reduce the cognitive load of the user without compromising route recognition. Besides navigation apps, other map-based LBS as point of interest locators may benefit from our results. However, further research concerning map reduction levels, completeness, landmark display, and their effects on orientation and navigation performance is required for gaining a deeper understanding of how to design task-oriented maps.

Author Contributions: Conceptualization, J.K., F.-B.M., D.E., F.D., and L.K.; Methodology, J.K. and L.K.; Formal Analysis, J.K. and L.K.; Investigation, J.K.; Resources, J.K.; Writing-Original draft preparation, J.K. and F.-B.M.; Writing-review and editing, F.-B.M., D.E., F.D., L.K., and J.K..; Visualization, J.K.; Project Administration, F.D. and L.K.; Funding Acquisition, F.D., L.K., F.-B.M., and D.E.

Funding: This study was supported by grants of the Deutsche Forschungsgemeinschaft (DFG) to FBM (FA 1189/3-1), FD (DI 771/11-1), and LK (KU 2872/6-1).

Conflicts of Interest: The authors declare no conflicts of interest. The founding sponsors had no role in the design of the study; in the collection, analyses, or interpretation of data; in the writing of the manuscript, and in the decision to publish the results.

\section{References}

1. Mocnik, F.-B.; Zipf, A.; Raifer, M. The OpenStreetMap Folksonomy and Its Evolution. Geo-Spat. Inf. Sci. 2017, 20, 219-230. [CrossRef]

2. Cipeluch, B.; Jacob, R.; Mooney, P.; Winstanley, A. Comparison of the accuracy of OpenStreetMap for Ireland with Google Maps and Bing Maps. In Proceedings of the Ninth International Symposium on Spatial Accuracy Assessment in Natural Resuorces and Enviromental Sciences; Höhle, J., Ed.; University of Leicester: Leichester, UK, 2010; pp. 337-340.

3. Haklay, M. How good is volunteered geographical information? A comparative study of OpenStreetMap and Ordnance Survey datasets. Environ. Plan. B Plan. Des. 2010, 37, 682-703. [CrossRef]

4. Brauen, G. Interactive Audiovisual Design for Cartography: Survey, Prospects, and Example. In Developments in the Theory and Practice of Cybercartography; Taylor, D.R.F., Lauriault, T.P., Eds.; Elsevier Science: Amsterdam, the Netherlands, 2014; Volume 5, pp. 141-159, ISBN 9780444627131.

5. Peterson, M.P. Elements of Multimedia Cartography. In Multimedia Cartography; Cartwright, W., Peterson, M.P., Gartner, G., Eds.; Springer: Heidelberg, Germany, 2007; pp. 64-73, ISBN 978-3-540-36650-8.

6. Taylor, D.R.F.; Lauriault, T.P. Future Directions for Multimedia Cartography. In Multimedia Cartography; Cartwright, W., Peterson, M.P., Gartner, G., Eds.; Springer: Heidelberg, Germany, 2007; pp. 505-522, ISBN 978-3-540-36650-8.

7. Mocnik, F.-B.; Fairbairn, D. Maps Telling Stories? Cartogr. J. 2018, 55, 36-57. [CrossRef]

8. Meilinger, T.; Hölscher, C.; Büchner, S.J.; Brösamle, M. How much information do you need? Schematic maps in wayfinding and self localisation. In Proceedings of the International Conference on Spatial Cognition V, Spatial Cognition 2006; Barkowsky, T., Knauff, M., Ligozat, G., Montello, D.R., Eds.; Lecture Notes in Computer Science; Springer: Berlin/Heidelberg, Germany, 2006; Volume 4387, pp. 381-400, ISBN 978-3-540-75665-1.

9. Thorndyke, P.W.; Hayes-Roth, B. Differences in Spatial Knowledge Acquired from Maps and Navigation. Cogn. Psychol. 1982, 14, 560-589. [CrossRef] 
10. Kuchinke, L.; Dickmann, F.; Edler, D.; Bordewieck, M.; Bestgen, A.K. The processing and integration of map elements during a recognition memory task is mirrored in eye-movement patterns. J. Environ. Psychol. 2016, 47, 213-222. [CrossRef]

11. Edler, D.; Bestgen, A.K.; Kuchinke, L.; Dickmann, F. Grids in Topographic Maps Reduce Distortions in the Recall of Learned Object Locations. PLoS ONE 2014, 9. [CrossRef]

12. Edler, D.; Dickmann, F.; Bestgen, A.K.; Kuchinke, L. The effects of grid line separation in topographic maps for object location memory. Cartogr. Int. J. Geogr. Inf. Geovisualization 2014, 49, 207-217. [CrossRef]

13. Edler, D.; Keil, J.; Bestgen, A.K.; Kuchinke, L.; Dickmann, F. Hexagonal Map Grids—An Experimental Study on the Performance in Memory of Object Locations. Cartogr. Geogr. Inf. Sci. 2018. [CrossRef]

14. Lee, H.; Plass, J.L.; Homer, B.D. Optimizing cognitive load for learning from computer-based science simulations. J. Educ. Psychol. 2006, 98, 902-913. [CrossRef]

15. Ishikawa, T.; Fujiwara, H.; Imai, O.; Okabe, A. Wayfinding with a GPS-based mobile navigation system: A comparison with maps and direct experience. J. Environ. Psychol. 2008, 28, 74-82. [CrossRef]

16. Parush, A.; Ahuvia-Pick, S.; Erev, I. Degradation in Spatial Knowledge Acquisition When Using Automatic Navigation Systems. In Proceedings of the 8th International Conference on Spatial Information Theory; Winter, S., Duckham, M., Kulik, L., Kuipers, B., Eds.; Springer: Berlin/Heidelberg, Germany, 2007; pp. 238-254, ISBN 9781608455959.

17. Münzer, S.; Zimmer, H.D.; Schwalm, M.; Baus, J.; Aslan, I. Computer Assisted Navigation and the Acquisition of Route and Survey Knowledge. J. Environ. Psychol. 2006, 26, 300-308. [CrossRef]

18. Wang, J.; Li, R. An empirical study on pertinent aspects of sketch maps for navigation. Int. J. Cogn. Inform. Nat. Intell. 2013, 7, 26-43. [CrossRef]

19. Wang, J.; Schwering, A. The Accuracy of Sketched Spatial Relations: How Cognitive Errors Affect Sketch Representation. In Proceedings of the International Workshop Presenting Spatial Information: Granularity, Relevance, and Integration; Tenbrink, T., Winter, S., Eds.; University of Melbourne: Bremen, Germany; Melbourne, Australia, 2009; pp. 41-56.

20. Blaser, A.D. A study of people's sketching habits in GIS. Spat. Cogn. Comput. 2000, 2, 393-419. [CrossRef]

21. Tversky, B.; Lee, P.U. Pictorial and Verbal Tools for Conveying Routes. In Proceedings of the International Conference on Spatial Information Theory; Freksa, C., Mark, D.M., Eds.; Springer: Berlin/Heidelberg, Germany, 1999; pp. 51-64, ISBN 978-3-540-66365-2.

22. Billinghurst, M.; Weghorst, S. The Use of Sketch Maps to Measure Cognitive Maps of Virtual Environments. In Proceedings of the Virtual Reality Annual International Symposium '95; Spencer Sipple, R., Ed.; IEEE: Los Alamitos, LA, USA, 1995; pp. 40-47, ISBN 0-8186-7084-3.

23. Robinson, A.H. Elements of Cartography; John Wiley \& Sons, Inc.: New York, NY, USA, 1953.

24. Colby, G.; Scholl, L. Transparency and blur as selective cues for complex visual information. In Image Handling and Reproduction Systems Integration; Bender, W.R., Plouffe, W., Eds.; SPIE: San Jose, CA, USA, 1991; Volume 1460, pp. 114-126.

25. Tsai, M.J.; Hou, H.T.; Lai, M.L.; Liu, W.Y.; Yang, F.Y. Visual attention for solving multiple-choice science problem: An eye-tracking analysis. Comput. Educ. 2012, 58, 375-385. [CrossRef]

26. Liu, H.; Heynderickx, I. Visual Attention in Objective Image Quality Assessment: Based on Eye-Tracking Data. IEEE Trans. Circ. Syst. Video Technol. 2011, 21, 971-982. [CrossRef]

27. Just, M.A.; Carpenter, P.A. Eye Fixations and Cognitive Processes. Cogn. Psychol. 1976, 8, 441-480. [CrossRef]

28. Steck, S.D.; Mallot, H.A. The Role of Global and Local Landmarks in Virtual Environment Navigation. Presence Teleoper. Virtual Environ. 2000, 9, 69-83. [CrossRef]

29. Golledge, R.G. Wayfinding Behavior: Cognitive Mapping and Other Spatial Processes; JHU Press: Baltimore, MD, USA, 1999.

30. Tom, A.; Denis, M. Language and Spatial Cognition: Comparing the Roles of Landmarks and Street Names in Route Instructions. Appl. Cogn. Psychol. 2004, 18, 1213-1230. [CrossRef]

31. Keil, J.; Edler, D.; Dickmann, F.; Kuchinke, L. Meaningfulness of Landmark Pictograms Reduces Visual Salience and Recognition Performance. Appl. Ergon. 2019, 75, 214-220. [CrossRef] [PubMed]

32. Caduff, D.; Timpf, S. On the assessment of landmark salience for human navigation. Cogn. Process. 2008, 9, 249-267. [CrossRef] 
33. Beaucousin, V.; Cassotti, M.; Simon, G.; Pineau, A.; Kostova, M.; Houdé, O.; Poirel, N. ERP evidence of a meaningfulness impact on visual global/local processing: When meaning captures attention. Neuropsychologia 2011, 49, 1258-1266. [CrossRef] [PubMed]

34. Nichols, A.L.; Maner, J.K. The Good-Subject Effect: Investigating Participant Demand Characteristics. J. Gen. Psychol. 2008, 135, 151-166. [CrossRef] [PubMed]

35. Orne, M.T. On the social psychology of the psychological experiment: With particular reference to demand characteristics and their implications. Am. Psychol. 1962, 17, 776-783. [CrossRef]

36. Nevin, J.A. Signal Detection Theory and Operant Behaviour: A Review of David M. Green and John A. Swets' Signal Detection Theory and Psychophysics. J. Exp. Anal. Behav. 1969, 12, 475-480. [CrossRef]

37. Harris, J. Sensation and Perception; SAGE: London, UK, 2014; ISBN 9780857020642.

38. Macmillan, N.A.; Creelman, C.D. Response Bias: Characteristics of Detection Theory, Threshold Theory, and "Nonparametric" Indexes. Psychol. Bull. 1990, 107, 401-413. [CrossRef]

39. Rayner, K. Eye movements and attention in reading, scene perception, and visual search. Q. J. Exp. Psychol. 2009, 62, 1457-1506. [CrossRef] [PubMed]

40. Bojko, A. Eye Tracking the User Experience: A Practical Guide to Research; Rosenfeld Media: New York, NY, USA, 2013; ISBN 1933820101.

41. Ziegler, A. Generalized Estimating Equations; Springer: Heidelberg, Germany, 2011.

42. O'Neill, M.J. Effects of signage and floor plan configuration on wayfinding accuracy. Environ. Behav. 1991, 23, 553-574. [CrossRef]

43. Franke, C.; Schweikart, J. Investigation of Landmark-Based Pedestrian Navigation Processes with a Mobile Eye Tracking System. In Progress in Location-Based Services 2016; Springer: Berlin/Heidelberg, Germany, 2017; pp. 105-130.

44. Dutta-Bergman, M.J. The Impact of Completeness and Web Use Motivation on the Credibility of e-Health Information. J. Commun. 2004, 54, 253-269. [CrossRef]

45. Bellezza, F.S.; Six, L.S.; Phillips, D.S. A mnemonic for remembering long strings of digits. Bull. Psychon. Soc. 1992, 30, 271-274. [CrossRef]

(C) 2018 by the authors. Licensee MDPI, Basel, Switzerland. This article is an open access article distributed under the terms and conditions of the Creative Commons Attribution (CC BY) license (http://creativecommons.org/licenses/by/4.0/). 